\title{
Uso del gel LAT para suturar heridas en niños
}

\author{
T. Valls Durána ${ }^{\mathrm{a}}$ E. Díaz Sanisidro ${ }^{\mathrm{b}}$, L. Nadal González ${ }^{\mathrm{b}}$ \\ aPediatra. Servicio de Pediatría. \\ ${ }^{b}$ MIR de Medicina de Familia. \\ CS Val Miñor. Nigrán, Pontevedra. España.
}

Rev Pediatr Aten Primaria. 2009; I 1:575-85

Teresa Valls Durán, teresa_valls@hotmail.es

\section{Resumen}

Introducción: las laceraciones traumáticas que precisan puntos de sutura son uno de los motivos de consulta urgente más frecuentes en pediatría. Conseguir la mejor anestesia de la zona causando el mínimo dolor posible es el paso previo y fundamental para una correcta reparación de la lesión en óptimas condiciones. El objetivo de este trabajo es comprobar la eficacia del gel anestésico LAT (lidocaína, 1,5\%; adrenalina, 0,1\%; y tetracaína, 1\%) para suturar las heridas no complicadas en pacientes pediátricos.

Material y métodos: fueron incluidos los pacientes pediátricos que acudieron a nuestro centro de salud con heridas para suturar desde julio de 2007 hasta diciembre del año siguiente. El dolor sentido se midió mediante la escala visual analógica (EVA) en los niños que podían expresarse verbalmente, y a través de la Face Pain Scale (FPS) en los más pequeños.

Resultados: se incluyó un total de 106 pacientes. La media de edad fue de 5,94 años, y la medida del dolor fue de 1,34 en el grupo valorado mediante EVA y de 1,67 en el de la FPS. La eficacia fue significativamente mayor cuando las heridas se situaban en la cara o el cuero cabelludo con respecto a las extremidades. No hubo complicaciones importantes (el seguimiento se llevó a cabo mediante una llamada telefónica) y los pacientes y sus familias quedaron satisfechos.

Conclusiones: la aplicación de gel LAT resulta útil para suturar heridas no complicadas en niños sin ocasionar dolor, especialmente cuando éstas se localizan en la cara y el cuero cabelludo.

Palabras clave: Anestesia tópica, Lidocaína, Tetracaína, Adrenalina, Laceraciones cutáneas, Suturas.

Abstract

Introduction: traumatic lacerations requiring suturing stitches are one of the most frequent reasons of emergencies in paediatrics. Achieving the best anesthesia of the area in order to cause the minimum pain possible is the previous and basic step to a successful repair of the injury under ideal conditions. The aim of this work is to check the LAT anesthetic gel efficacy (lidocaine, 1.5\%; adrenalin, 0.1\%; tetracaine, 1\%) in order to suture no complicated wounds in paediatric patients.

Los autores declaran no presentar conflictos de intereses en relación con la preparación y publicación de este artículo. 
Material and methods: paediatric patients coming to our health centre to get their wounds sutured from July 2007 to December 2008 were included. The evaluation of the pain felt was measured by using the Visual Analogue Scale (VAS) in those children who could express themselves verbally and by the Face Pain Scale (FPS) in the younger ones.

Results: a total of 106 patients were included. Mean age was 5.94 years, and the measurement of pain 1.34 in the group evaluated by VAS, and 1.67 in the one evaluated by FPS. Effectiveness was significantly higher with wounds located on face or scalp compared to those in the extremities. There were no major complications (monitored by phone call) and patients and their families seemed to be pleased.

Conclusions: LAT gel application is useful for suturing no complicated wounds in children without causing pain, especially with wounds located on face and scalp.

Key words: Topical anesthetics, Lidocaine, Tetracaine, Adrenaline, Skin lacerations, Sutures.

\section{Introducción}

La incorporación de la anestesia a la práctica quirúrgica es, sin duda, uno de los logros más importantes de la historia de la medicina. Los profesionales sanitarios tenemos la obligación ética de evitar el sufrimiento y el dolor del paciente en todas nuestras actuaciones y cuidados, y este grupo de fármacos nos ofrece las herramientas necesarias para tratar de conseguirlo.

Aunque la capacidad que tienen algunas sustancias naturales de interrumpir de forma temporal y reversible la transmisión nerviosa en la zona donde se aplican y de producir insensibilidad se conoce desde hace siglos -los incas lo comprobaban al mascar coca-, no fue hasta la segunda mitad del siglo XIX cuando se aisló por primera vez el principio activo de esta planta, la cocaína, y se empezaron a estudiar sus propiedades, sus efectos terapéuticos y su aplicación en medicina. Pero habían de pasar todavía varias décadas (1947) hasta que se sintetizara la primera sustancia de este grupo, la lidocaína, que sentaría las bases de la anestesia local. Éste es el primero de una serie de fármacos que se irán enriqueciendo y perfeccionando con el tiempo.

En la actualidad, la lidocaína al 1-2\% infiltrada sigue siendo el método de anestesia estándar para múltiples procedimientos quirúrgicos que requieren anestesia local. Es un fármaco versátil, seguro, eficaz, rápido, de duración moderada pero suficiente, y que cuenta con una larga experiencia de uso. Pero presenta un inconveniente: el dolor causado durante su administración, especialmente en los niños, en quienes este hecho suele adquirir una relevancia 
mayor pues son especialmente lábiles al componente emocional del dolor y comprenden con dificultad la necesidad de cooperación que se requiere en estos casos.

Tratando de paliar esta desventaja se empezó a desarrollar la anestesia tópica, que consiste en la aplicación en distintas proporciones de fórmulas magistrales compuestas por anestésicos locales, generalmente asociados a vasoconstrictores. Éstas, aplicadas sobre la piel -íntegra en unos casos o abierta en otros-, permiten mitigar el dolor causado en procedimientos diagnósticos o terapéuticos, sin necesidad de recurrir a la infiltración con la agresión adicional que ello supone.

El objetivo de este trabajo es comprobar en nuestro medio el efecto anestésico del gel LAT según la fórmula propuesta por un grupo de Alicante1 (lidocaína, 1,5\%; adrenalina, 0,1\%; y tetracaína $1 \%)^{1}$ para suturar las heridas no complicadas en pacientes pediátricos.

\section{Material y métodos}

Se incluyó a todos los pacientes de 115 años que acudieron al centro de salud o al servicio de urgencias desde julio de 2007 hasta diciembre del año siguiente, con heridas simples que cum- plían los criterios de inclusión y que precisaban puntos de sutura.

Criterios de inclusión: pacientes mayores de 1 año con heridas no complicadas en partes no distales del cuerpo (puntas de los dedos, pene, pabellón auricular, nariz, etc.).

Criterios de exclusión: pacientes inconscientes o con el sensorio alterado; heridas en partes acras; heridas en mucosas; heridas profundas que involucraran hueso, cartílago, tendones o vasos importantes; heridas mayores de $6 \mathrm{~cm}$ de longitud; heridas muy sucias o contaminadas; heridas de más de 3 horas de evolución; alergia conocida a algún anestésico local; y no autorización expresa del paciente o del padre, la madre o el tutor.

Se informó al paciente o al padre, la madre o el tutor y le fue solicitado el consentimiento informado.

El gel fue elaborado por una farmacéutica de la zona según la fórmula proporcionada por el Centro de Información del Medicamento del Colegio de Farmacéuticos de Pontevedra.

El tratamiento se llevó a cabo en todo momento en condiciones de asepsia: se limpiaba debidamente la herida con suero, se aplicaba una capa de gel de unos $5 \mathrm{~mm}$ por encima de la herida abarcando y rebasando los bordes, se 
cubría con una gasa y se dejaba durante 20 minutos. Después de este tiempo se comprobaba el dolor con una aguja: si había sido eficaz, se retiraba el gel con una gasa y se procedía a la sutura. Si la zona seguía estando sensible, se aplicaba una nueva capa de gel y se esperaba 10 minutos más. Si después de este tiempo persistía el dolor, se ofrecía la posibilidad de infiltrar con lidocaína.

Los datos del paciente y de su lesión quedaban registrados en una hoja, donde también se hacía constar el número de aplicaciones del gel, el tiempo requerido, así como la valoración del dolor sentido y evaluado por el paciente mediante la escala analógica visual (EVA), en el caso de que pudiera expresarlo verbalmente, o percibido de forma indirecta por los familiares y/o el profesional en el niño pequeño que no podía puntuarlo de dicha forma. En este último caso, se intentaba captar mediante la Face Pain Scale (FPS) el dolor causado por la propia sutura, tratando de desligarlo del llanto continuo, que no se modificaba ni aumentaba de intensidad en el momento de la punción, derivado de la angustia habitual en los niños pequeños ante estas situaciones.

Finalmente, se llevó a cabo un seguimiento posterior mediante una llamada telefónica con el fin de detectar posibles complicaciones y conocer el grado de satisfacción.

Para analizar las diferencias en la respuesta dolorosa entre suturar en la cabeza o en las extremidades, se aplicó el test de Kruskal-Wallis de comparación de medias. Se escogió esta prueba no paramétrica debido al bajo número de heridas en las extremidades (se aplicó la EVA en 17 casos y la FPS sólo en 6). Se empleó el método de regresión lineal para correlacionar los valores obtenidos en las escalas EVA y FPS con dos variables -la edad del paciente y la longitud del corte-, buscando una relación entre ellas. Asimismo, se utilizó el test de análisis de varianzas (ANOVA) para estudiar las diferencias entre la edad de los pacientes y la localización de los cortes.

\section{Resultados}

Se registró un total de 116 pacientes, recogidos de forma consecutiva. De éstos, 108 fueron incluidos en el análisis estadístico y 8 se descartaron por la recogida incompleta de los datos.

En la tabla I se muestra un resumen de los estadísticos descriptivos hallados. Desde el momento en que se produjeron los cortes, se tardó en recibir atención médica una media de $34 \mathrm{mi}$ nutos, con una desviación estándar de 
28 minutos, a los que hay que sumar los 20 minutos de aplicación del gel LAT para iniciar la sutura. Sólo en dos pacientes cuyas heridas se localizaban en las extremidades inferiores se completó la anestesia con una infiltración de lidocaína.

En la tabla II se muestran las diferencias de la respuesta al dolor en función de la localización de la herida. Como puede comprobarse, la eficacia fue significativamente mayor en las heridas situadas en la cabeza, tanto para los casos medidos por EVA como por FPS.

Ninguna de las rectas calculadas para correlacionar los valores obtenidos en las escalas EVA y FPS con dos variables como la edad del paciente y la longitud del corte mediante regresión lineal aportó una significación estadística suficiente ( $p>0,05$ en todos los casos). Tampoco los coeficientes de Pearson fueron relevantes, por lo que estas variables no parecen explicar en absoluto la respuesta al dolor y se podrían considerar independientes de ella. En la tabla III se muestra un resumen de este análisis de correlación bivariada.

Se observó una diferencia significativa de edad entre los pacientes con cortes en las extremidades (que eran mayores) y los niños con cortes en cabeza (que resultaron ser más pequeños). También fue significativamente menor la edad

Tabla I. Estadísticos descriptivos de la muestra

\begin{tabular}{|c|c|c|}
\hline$N$ & & 108 \\
\hline Edad media $\pm \mathrm{DE}$ & & $5,94 \pm 3,70$ años \\
\hline Localización de las heridas & Cabeza & $85(78,7 \%)$ \\
\hline & Extremidades & $23(21,3 \%)$ \\
\hline Longitud media de los cortes \pm & & $2,35 \pm 1,14 \mathrm{~cm}$ \\
\hline Pacientes a los que se aplicó la & & $62(57,4 \%)$ \\
\hline Pacientes a los que se aplicó la & & $46(42,6 \%)$ \\
\hline Respuesta media al dolor $\pm \mathrm{DE}$ & EVA & $1,34 \pm 2,35$ \\
\hline & FPS & $1,67 \pm 1,70$ \\
\hline
\end{tabular}

Tabla II. Diferencias en la respuesta al dolor respecto a la localización de la herida

\begin{tabular}{|c|c|c|c|c|c|c|}
\hline & \multicolumn{2}{|c|}{ Heridas en la cabeza } & \multicolumn{4}{|c|}{ Heridas en las extremidades } \\
\hline & $\mathrm{n}$ & Media $\pm \mathrm{DE}$ & $\mathrm{n}$ & Media $\pm \mathrm{DE}$ & Significación (p) & Estadístico H \\
\hline EVA $(n=62)$ & 45 & $0,91 \pm 2,28$ & 17 & $2,31 \pm 2,21$ & 0,0002 & 13,7576 \\
\hline $\operatorname{FPS}(n=46)$ & 40 & $1,38 \pm 1,56$ & 6 & $3,67 \pm 1,21$ & 0,0028 & 8,9253 \\
\hline
\end{tabular}


media en la escala FPS que en la EVA. Los cálculos estadísticos se muestran en la tabla IV.

Por último, en la tabla $\mathrm{V}$ se recogen los resultados de la encuesta telefónica para conocer la evolución y el grado de satisfacción.

\section{Discusión}

Durante mucho tiempo, el dolor infantil ha sido infravalorado $y$, en consecuencia, infratratado. Se consideraba que el niño era incapaz de sentir dolor físico de la misma manera que el adulto, que las fibras de transmisión del impulso doloroso en esta edad estaban insuficientemente mielinizadas. Actualmente se sabe no solo que esto no es cierto, sino que el paciente pediátrico podría tener incluso un umbral para el dolor más bajo que el del adulto.

Por otro lado, está demostrado que el miedo ocupa una parte fundamental en la experiencia del dolor, sobre todo en lo que se refiere a procedimientos diagnósticos o terapéuticos; cuanto más asustado esté un paciente, más intensa y molesta será la sensación de dolor experimentado.

El hecho de que el niño sea especialmente vulnerable a estas vivencias (miedo a situaciones extrañas, a ser separado de sus seres queridos), unido a la constatación de que las fibras de

Tabla III. Significación estadistica de los análisis y las correlaciones de las variables

\begin{tabular}{l|l|l|l|l|l|l}
\hline & Variables & $\begin{array}{l}\text { Significación } \\
(\mathrm{p})\end{array}$ & $\begin{array}{l}\text { Coeficiente } \mathrm{r}^{2} \\
\text { Pearson }\end{array}$ & Estadístico $\mathrm{F}$ & Constante & Parámetro b1 \\
\hline EVA $(\mathrm{n}=62)$ & Longitud & 0,547 & 0,006 & 0,367 & 1,020 & 0,147 \\
& Edad & 0,576 & 0,005 & 0,315 & 1,710 & $-0,048$ \\
\hline \multirow{2}{*}{ FPS $(\mathrm{n}=46)$} & Longitud & 0,342 & 0,021 & 0,923 & 1,069 & 0,285 \\
\cline { 2 - 6 } & Edad & 0,391 & 0,017 & 0,752 & 1,318 & 0,102 \\
\hline
\end{tabular}

Tabla IV. Diferencias en la edad según la localización de la herida y la escala empleada

\begin{tabular}{|c|c|c|c|c|c|c|}
\hline & $\mathrm{He}$ & en la cabeza & $\mathrm{HeI}$ & s en extremidades & & \\
\hline & $n$ & Media $\pm \mathrm{DE}$ & $n$ & Media $\pm \mathrm{DE}$ & Significación (p) & Estadístico T \\
\hline Edad & 85 & $5,38 \pm 3,73$ & 23 & $8,04 \pm 2,75$ & 0,0018 & 3,199 \\
\hline & $\mathrm{Pac}$ & es EVA & $\mathrm{Pac}$ & tes FPS & Significación (p) & Estadístico $\mathrm{H}$ \\
\hline & $\mathrm{n}$ & Media $\pm \mathrm{DE}$ & $n$ & Media $\pm \mathrm{DE}$ & & \\
\hline Edad & 62 & $7,76 \pm 3,56$ & 46 & $3,50 \pm 2,17$ & $<0,0001$ & 40,655 \\
\hline
\end{tabular}


transmisión de los impulsos dolorosos están perfectamente desarrolladas desde los primeros días de vida, y a que las experiencias de dolor sentido hacen que descienda el umbral del dolor ulterior e incluso perturben el desarrollo en algunos aspectos, hace que en los últimos años haya surgido un creciente interés por el estudio del dolor en el niño, de los métodos para su diagnóstico y valoración, así como de su tratamiento.

En esta línea se han desarrollado fármacos y técnicas no farmacológicas con el objetivo de evitar, en la medida de lo posible, el dolor, tanto el derivado de la propia enfermedad o trastorno que lo ocasiona como el que se infringe en el curso de exploraciones diagnósticas o tratamientos agresivos. El empleo del gel LAT puede contribuir a lograr este objetivo.

De acuerdo con nuestra experiencia, el gel LAT, según la fórmula usada en este estudio, ha proporcionado una anestesia adecuada durante el procedimiento de reparación de las heridas me- diante sutura en los pacientes descritos, tal como lo acreditan las puntuaciones en cada una de las escalas utilizadas, con una media inferior a 2 (se considera que una anestesia es adecuada si el valor de la EVA es menor de 3).

Se trata de un estudio descriptivo $y$, como tal, no se ha comparado con otros grupos control tratados con otros métodos. Su objetivo ha sido verificar en nuestro medio la utilidad de una de las fórmulas de anestesia tópica ya estudiadas en pacientes pediátricos mediante ensayos clínicos publicados en las últimas décadas, para contribuir a su recomendación y difusión.

En 1980 se publica un primer ensayo clínico² que compara la eficacia de la lidocaína infiltrada con la fórmula anestésica TAC (tetracaína, 0,5\%; adrenalina, 1/2.000; y cocaína, 11,8\%) para aplicar de forma tópica en laceraciones simples de la cara y el cuero cabelludo de niños que acudían al servicio de urgencias. Los autores concluyen que la fórmula TAC es tan efectiva como la li-

Tabla V. Seguimiento de la evolución. Encuesta de satisfacción.

\begin{tabular}{l|l|l|l}
\hline & $\mathbf{n}$ & Porcentaje & Sobre el total \\
\hline Llamadas exitosas & 70 & $100 \%$ & $65 \%$ \\
\hline Complicaciones & 4 & $5,7 \%$ & $3,7 \%$ \\
\hline Problemas con sutura & 4 & $5,7 \%$ & $3,7 \%$ \\
\hline Satisfacción global & 66 & $94,3 \%$ & $61,1 \%$ \\
\hline ¿Volvería a utilizarlo? & 65 & $92,9 \%$ & $60,2 \%$ \\
\hline
\end{tabular}


docaína infiltrada para conseguir la anestesia de la zona, pero es mucho mejor aceptada. Este preparado presenta otras ventajas: no distorsiona los bordes de la herida, lo que hace más fácil la reparación, y se evita la posibilidad de punción accidental del profesional, rara pero posible.

Sin embargo, la presencia de cocaína, que es una sustancia obviamente arriesgada desde el punto de vista toxicológico, y la asociación de ésta a efectos secundarios graves, como síntomas neurológicos, convulsiones e incluso un caso de muerte atribuido a su absorción por vía mucosa, junto con el precio elevado que supone su presencia, hacen que el preparado pierda aceptación. Además se encontró cocaína en el plasma de niños tratados con la fórmula TAC. Algunos ensayos ${ }^{3}$ de formulaciones con menor cantidad de cocaína ( $4 \%$ versus $11,8 \%$ ) encontraron una eficacia idéntica pero los mismos problemas tóxicos y legales.

En 1995 aparecen dos trabajos -uno realizado en niños ${ }^{4}$ y otro en adultos ${ }^{5}-$ que estudian la eficacia de un nuevo preparado -LET (lidocaína, 4\%; epinefrina, 1/2.000; y tetracaína, 1\%)-, y lo comparan con la fórmula TAC. Ambos concluyen que LET es igual de eficaz pero más seguro, carece de efectos indeseables y es mucho más barato.
Desde entonces han sido varios los autores que han publicado sobre el tema, buscando otras alternativas:

- 1996: comparan TAC con TLE (lidocaína, 5\%, y epinefrina, 1/2.000) y no encuentran diferencias 6 .

- 1996: descubren que el Bupivanor $^{\circledast}$ (bupivacaína, 0,48\%, y norepinefrina, 1/26.000) es tan eficaz como TAC o lidocaína infiltrada 7 .

- 1997: comparan el Mepivanor ${ }^{\circledR}$ (mepivacaína, 2\%; norepinefrina, 1/100.000) con TAC y encuentran que el primero es menos efectivo que el segundo.

- 1997: comparan TAC con EMLA (mezcla eutéctica de prilocaína 25 $\mathrm{mg} / \mathrm{ml}$ + lidocaína $25 \mathrm{mg} / \mathrm{ml}$ ) para anestesiar laceraciones en extremidades en niños y concluyen que $E M L A^{\circledR}$ es superior a TAC y que las normas debían ser revisadas para que $E M L A^{\oplus}$ fuese aprobada para este tipo de tratamientos ${ }^{9}$.

Hasta el momento, EMLA ${ }^{\oplus}$ sólo tiene autorizado en ficha técnica su uso para pieles intactas $y$, aunque haya sido empleada en algunos trabajos, no existe experiencia en heridas y no se aconseja.

- 1998: comparan prilocaína-fenilefrina con bupivacaína-fenilefrina y con TAC. Concluyen que ambas preparaciones son efectivas pero 
que la prilocaína (Prilophen) es más eficaz que la bupivacaína ${ }^{10}$.

- 1998: comparan un preparado LET en solución con otro en gel y encuentran ventajas a favor de este último" ${ }^{11}$.

- 2001: comparan LET y EMLA ${ }^{\oplus}$ en el pretratamiento de laceraciones antes de infiltrar con lidocaína y concluyen que la eficacia es similar. Hay que puntualizar que es necesario esperar 90 minutos para observar el efecto de EMLA ${ }^{\oplus 12}$.

- 2001: Chipont y cols ${ }^{1}$, del Hospital San Juan de Alicante, evalúan la eficacia de una nueva formulación de LAT (lidocaína 1,5\% + adrenalina 1/1.000 + tetracaína 1\%) en laceraciones simples de la piel cercanas al ojo y concluyen que, en estas proporciones, LAT suministra una anestesia adecuada y debería ser tenido en cuenta en este tipo de lesiones.

A partir de toda la bibliografía revisada debe hacerse una serie de consideraciones:

- Todos estos anestésicos tópicos son útiles, sobre todo en laceraciones simples, que no excedan determinadas dimensiones $(6-8 \mathrm{~cm})$, localizadas en la cara y el cuero cabelludo, pues en estas zonas el te- jido está más vascularizado y es más receptivo a este tipo de anestesia. Algunos autores recomiendan utilizarlo en otras partes del cuerpo. Aunque su eficacia es menor, podría ser útil para aminorar el dolor de una posterior infiltración.

- Parece que la presencia de adrenalina (o algún vasoconstrictor análogo) es fundamental: impide o dificulta la absorción de los anestésicos, lo cual permite administrar dosis más altas, $y$, por tanto, logra una mayor eficacia sin riesgo, se prolonga la duración del efecto y es hemostático (este dato se evidencia por el halo blanquecino que aparece poco después de su aplicación), lo que ayuda a contener posibles hemorragias. Tiene el inconveniente de que no puede usarse en partes acras (puntas de los dedos, pene, pabellón auricular, nariz, etc.), si bien es verdad que existen opiniones encontradas en esta cuestión y algunos autores lo han utilizado sin complicaciones isquémicas de ningún tipo ${ }^{13}$.

- Son formulaciones magistrales que no están comercializadas. Necesitan que un farmacéutico las elabore. 
- Y un dato curioso: hasta hace poco, la práctica totalidad de los trabajos publicados procedía de EE. UU., Canadá y Australia. Las dudas sobre su eficacia, la falta de experiencia en su uso, el tener que elaborarse como fórmula magistral, la creencia de que sólo la lidocaína infiltrada es realmente útil o las connotaciones negativas que se asociaron a las presentaciones que contenían cocaína, pueden ser algunos de los motivos de la reticencia a incorporar este preparado en los servicios de urgencia de otros países. En los últimos años parece que esto está cambiando $y$, concretamente en nuestro país, algunos autores están empezando a comunicar su experiencia ${ }^{14,15}$.

\section{Conclusiones}

El tratamiento del dolor infantil en todos sus aspectos ha de ser una prioridad para el pediatra.

El gel LAT es útil para suturar las heridas simples de los niños, sobre todo cuando éstas se localizan en la cara o el cuero cabelludo, ya que minimiza el dolor que comporta este procedimiento. Debería ser accesible y formar parte del botiquín de todos los centros de salud.

\section{Agradecimientos}

Los autores agradecen a Sonsoles $\mathrm{Ni}$ mo Sotelo su ayuda en la búsqueda de documentación y elaboración de la fórmula, y a los profesionales sanitarios del PAC Val Miñor por su colaboración en la recogida de los datos.

\section{Bibliografía}

1. Chipont Benavent E, García-Hermosa P, Alió Sanz JL. Sutura de laceraciones cutáneas con gel LAT (lidocaína, adrenalina, tetracaína). Arch Soc Esp Oftalmol. 2001;76:505-8.

2. Pryor GJ, Kilpatrick WR, Opp DR. Local anesthesia in minor lacerations: topical TAC versus lidocaine infiltration. Ann Emerg Med. 1980;9: 568-71.

3. Vinci BJ, Fish SS. Efficacy of topical anesthesia in children. Arch Pediatr Adolesc Med. 1996;150: 466-9.

4. Schilling CG, Bank DE, Borchert BA, Klatzko
MD, Uden DL. Tetracaine, epinephrine (adrenalin), and cocaine (TAC) versus lidocaine, epinephrine, and tetracaine (LET) for anesthesia of lacerations in children. Ann Emerg Med. 1995; 25(2):203-8.

5. Ernst AA, Marvez-Valls E, Nick TG, Weiss SJ. LAT (lidocaine-adrenaline-tetracaine) versus TAC (tetracaine-adrenaline-cocaine) for topical anesthesia in face and scalp lacerations. Am J Emerg Med. 1995;13:151-4.

6. Blackburn PA, Butler $\mathrm{KH}$, Hugues MJ, Clark MR, Riker RL. Comparison of tetracaine-adrenaline-cocaine (TAC) with topical lidocaine-epinephrine (TLE): efficacy and cost. Am J Emerg Med. 1995;13:315-7. 
7. Smith GA, Strausbaugh SD, Herbeck-Weber C, Shields BJ, Powers JD, Hackenberg D. Comparison of topical anesthetics without cocaine to tetracaine-adrenaline-cocaine and lidocaine infiltration during repair of lacerations: bupivacaine-norepinephrine is an effective new topical anesthetic agent. Pediatrics. 1996; 97:301-7.

8. Smith GA, Strausbaugh SD, Harbeck-Weber C, Cohen DM, Shields BJ, Powers JD. New non-cocaine-containing topical anesthetics compared with tetracaine-adrenaline-cocaine during repair lacerations. Pediatrics. 1997;100:825-30.

9. Zemsky WT, Karasic RB. EMLA versus TAC for topical anesthesia of extremity wounds in children. Ann Emerg Med. 1997;30:163-6.

10. Smith GA, Strausbaugh SD, Harbeck-Weber $C$, et al. Prilocaine-phenylephrine and bupivacaine-phenylephrine topical anesthetics compared with tetracaine-adrenaline-cocaine during repair of lacerations. Am J Emerg Med. 1998;16:121-4.

11. Resh $K$, Schilling C, Borchert BD, Klatzko
M, Uden D. Topical anesthesia for pediatric lacerations: a randomized trial lidocaine-epinephrine-tetracaine solution versus gel. Ann Emerg Med. 1998;32:693-7.

12. Singer AJ, Stark MJ. LET versus EMLA for pretreating lacerations: a randomized trial. Acad Emerg Med. 2001;8:223-30.

13. White NJ, Kim MK, Brousseau DC, Bergholte J, Hennes $\mathrm{H}$. The anesthetic effectiveness of lidocaine-adrenaline-tetracaine gel of finger lacerations. Pediatr Emerg Care. 2004;20:812-5.

14. Muga O, Arroabarren E, Rezola E, Hernández U, Muñoz JA. Eficacia del gel LAT como anestésico en la reparación de heridas mediante sutura. XII Reunión de la Sociedad Española de Urgencias Pediátricas. San Sebastián-Donostia, abril 2007.

15. Luaces $C$, Fernández Y. Avanzando en el manejo del dolor en Pediatría. XXII Congreso Nacional de la Sociedad Española de Pediatría Extrahospitalaria y Atención Primaria. Tenerife, octubre 2008. 